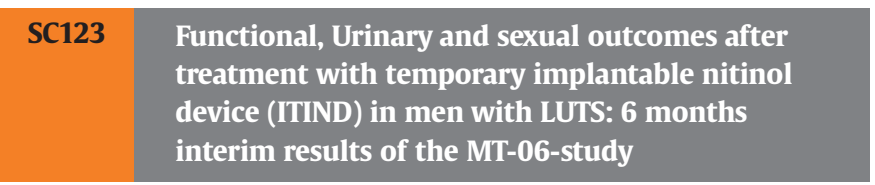

C. De Nunzio, V. Baldassarri, F. Cantiello, F. Crocerossa, C. Fiori, D. Amparore, F. Porpiglia, P. Tognoni, J. Reinoso Elbers, F. Gomez Sancha (Roma)

Introduction: To evaluate the functional and sexual outcomes after treatment with temporary implantable nitinol device (iTind; MediTate Ltd, Israel); a novel minimally-invasive treatment for Lower Urinary Tract Symptoms (LUTS)due to Benign Prostatic Hyperplasia (BPH).

Materials and methods: Following EC approval, 70 men with symptomatic BPH (IPSS $\geq 10$, Qmax $<12 \mathrm{ml} / \mathrm{sec}$, and prostate volume $(\mathrm{PV})<120 \mathrm{ml}$ ) were enrolled in a single arm, prospective multicenter study (MT06). Patients with a large median lobe $(>12 \mathrm{~mm})$, previous prostatic surgery, neurological dysfunction and urinary tract infections were excluded. Patients were not washed out of drug therapy before the procedure. I-TIND was implanted through a cystoscopy under sedation and it was removed 5-7 days later under local anesthesia. Post-operative VAS, QoR VAS, complications (Clavien Dindo-Grading System) were recorded. Preservation of urinary continence and sexual and ejaculatory function were assessed according to ISI, MSHQ-EjD and SHIM. Post-operative IPSS, QL, Qmax were also assessed at 1,3, and 6 months post-operatively.

Results: Overall 70 patients were enrolled, median age was $62.31 \mathrm{yrs}$, mean PV $37.68 \mathrm{ml}(15-80 \mathrm{ml})$. Baseline and follow-up data are reported in Table 1 . No intraoperative complications were observed and the average post-operative VAS score was $3.24 \pm 2.56$. On average patients returned to daily life after 4.3 days following the retrieval procedure, and mean QoR VAS was 0.77. Significant improvements from baseline levels were recorded in IPSS $(25.28 \pm 6.53$ vs $10.27 \pm 7.86$, $\mathrm{p}<0.0001)$, QoL $(4.13 \pm 1.01 \mathrm{vs} 1.96 \pm 1.45, \mathrm{p}<0.0001)$ and peak flow $(7.34 \pm 2.22$ vs $12.08 \pm 5.35, \mathrm{p}<0.0001)$ in response to iTind treatment at 6 months, and sexual and urinary continence were preserved.

Conclusions: iTind is a well-tolerated, minimally-invasive treatment for BPH-related LUTS offering a rapid recovery and return to daily life, preservation of sexual function and urinary continence as well as a significant improvement of symptoms and urinary flow at 6 months follow up.

\section{SC124 Predicting factors of post-operative acute urinary retention after Greenlight laser photoselective vaporization of the prostate}

D. Campobasso, A. Acampora, C. De Nunzio, F. Greco, M. Marchioni, P. Destefanis, V. Altieri, F. Bergamaschi, G. Fasolis, F. Varvello, S. Voce, F. Palmieri, C. Divan, G. Malossini, R. Oriti, L. Ruggera, A. Tuccio, A. Tubaro, G. Delicato, A. Laganà, C. Dadone, L. Pucci, M. Carrino, F. Montefiore, S. Germani, R. Miano, S. Rabito, G. De Rienzo, A. Frattini, G. Ferrari, L. Cindolo (Guastalla)

Introduction: Greenlight photoselective vaporization of the prostate (PVP) is a mini-invasive technique to treat Benign Prostatic Obstruction (BPO). Some of the advantages of Greenlight are shorter catheterization time and hospital stay compared to TURP. Postoperative acute urinary retention ( pAUR) leads to patients' discomfort, prolonged hospital stay and increased health care costs. We analyzed the risk factors for urinary retention after GreenLight laser PVP.

Materials and methods: In a multicenter experience, we retrospectively analyzed the onset of post-operative acute urinary retention in patients undergoing standard or anatomical PVP. The pre-, intra- and post-operative characteristics were compared between the patients who started to void and the patients who developed pAUR.

RESULTS: The multicenter database was composed of 1077 patients, but only 434 were suitable for the study. Post-operative acute urinary retention occurred in 39 patients (9\%). At the univariate analysis history of preoperative urinary retention $(p=0.217)$, age $(p=0,359)$, IPSS score $(p=0,132)$, surgical techniques (standard or anatomical, $\mathrm{p}=0,464)$, operative time $(\mathrm{p}=0,314)$, energy used and density ( $p=0,148$ and 0,573 ) and type of anesthesia (general or spinal, $\mathrm{p}=0,574$ ) did not differ between the two groups. A lower prostate volume was present in patients which developed pAUR (45 ml IQR 3555 vs $58.5 \mathrm{ml}$ IQR 45-74, p < 0.001). Patients with an adenoma volume less than $40 \mathrm{ml}$ had a higher probability to develop pAUR at univariate analyses $(82.1 \%, \mathrm{p}<0.001)$ and the type of the pre-operative medical treatment for BPO was linked to this risk ( $p=0,037$ ). The multivariate logistic regression showed that an IPSS $\geq 19$ was associated with a higher probability to develop pAUR (OR $=3,58$; 95\%CI 1,19-10,77; $\mathrm{p}=0,023$ ). Lower lasing time has resulted at the univariate analysis, correlated to the incidence of pAUR $(p=0.013)$. This data was confirmed at the multivariate logistic regression $(\mathrm{OR}=0,93 ; 95 \% \mathrm{Cl}$ $0,86-0,99 ; p=0.046$ ). The age adjusted logistic regression showed that the higher was the adenoma volume the lower was the probability to develop a pAUR. Furthermore, an increase in adenoma volume of $1 \mathrm{ml}$ was associated with a decrease of the probability of pAUR of $7 \%$ $(\mathrm{OR}=0.93 ; 95 \% \mathrm{CI} 0.89-0.98 ; \mathrm{p}=0.004)$. The multivariate logistic regression has confirmed that energy used and the operative time did not influence the incidence of pAUR $(p=0.141$ and 0.190 , respectively). In addition, the difference between the two groups in terms of medical treatments have preserved their significance. Patients treated with 5 alpha-reductase inhibitors (5-ARI) reported a higher probability of pAUR than those treated with alpha-blockers or no treatments $(\mathrm{OR}=4.01 ; 95 \% \mathrm{CI} 1.06-15.19 ; \mathrm{p}=0.041)$.

Conclusions: In our series, pAUR was related to low adenoma volume and lasing time, pre-operative IPSS $\geq 19$ and 5ARI intake. These data should be considered in considering the best timing for urethral catheters removal after Greenlight PVP.

\section{SC125 Prospective multicentre randomized study to compare holmium laser enucleation of prostate and laparoscopic/robot-assisted simple prostatectomy in high volume prostate glands $(\geq 120 \mathrm{ml})$}

A. Fuschi, Y. Al Salhi, A. Martoccia, S. Scalzo, G. Velotti, L. Capone, P. P. Suraci, A. Carbone, F. Annino, K. Saba, A. Asimakopoulos, G. Bozzini, M. Falsaperla, A. L. Pastore (Latina)

Introduction: The aim of this prospective randomized study is to compare the perioperative and functional results between laparoscopic and robot-assisted simple prostatectomy sec. Millin (LSP and RASP) and Holmium laser enucleation of prostate (HoLEP) in prostate volumes $\geq 120 \mathrm{ml}$. The primary endpoint will be to investigate whether the prostate gland volume may influence perioperative and functional outcomes, and the secondary endpoint will evaluate the frequency and type of postoperative complications according to Clavien Dindo classification.

Materials and methods: This multicentric study was conducted prospectively, from January 2016 to July 2018, on male patients with LUTS associated with BPH candidates for surgical treatment. The surgery approach choice in relation to the prostatic volume $\geq 120 \mathrm{ml}$ was HoLEP or minimally invasive simple prostatectomy (LSP or RASP) with extraperitoneal access as originally described by Millin. All patients, candidates for prostate surgery in accordance with the European Association of Urology (EAU) guidelines, were prospectively 
randomized into three groups, according to a simple computed randomization: HoLEP, LSP and RASP groups. During the follow-up, all patients underwent post-operative control at 1, 3, 6, 12 and 24 months from the surgical procedure.

Results: 110 male patients were grouped in three homogeneous groups: 36 patients in LSP group, 32 in RASP one and 42 in HoLEP group. During the follow-up (mean 26.15 months), despite the significant improvement compared to baseline results, no significant differences were shown, between the groups, in relation to: operative time ( $p$ value: 0.175 ), drop of Hemoglobin ( $p$ value: 0.271 ), residual prostate volume ( $\mathrm{p}$ value: 0.122 ), surgical complication in accordance to Clavien Dindo classification ( $p$ value $>0.05$ ), uroflowmetric parameters ( $p$ value $>0.05$ ), post void residual volume ( $p$ value: 0.09 ), international prostate symptom score (IPSS; $\mathrm{p}$ value: 0.863 ), international index of erectile function (IIEF-5; p value: 0.185). The catheterization time was statistically longer in the LSP group than RASP and HoLEP groups ( $p$ value: 0.002 ). During the first month of follow-up, a higher percentage of patients with new-onset irritative symptoms were recorded in the HoLEP group than LSP and RASP groups, after completely resolved, also by medical therapy.

Conclusions: This prospective randomized study is the first to compare extraperitoneal LSP, RASP and HoLEP in the treatment of LUTS secondary to benign prostatic hyperplasia for prostate volumes $\geq 120 \mathrm{ml}$. The study confirms the safety and efficacy of minimally invasive techniques in terms of peri and postoperative and functional outcomes comparable to HoLEP.

SC126 Sexual and functional outcomes of prostate artery
embolisation: a prospective long-term follow-up,
large cohort study

L. Capone, A. Fuschi, G. Velotti, A. Martoccia, P. P. Suraci, S. Scalzo, A. Carbone, E. Illiano, E. Costantini, Y. Al Salhi, A. L. Pastore (Latina)

Introduction: Among minimally invasive procedures for treating benign prostate hyperplasia (BPH) prostate artery embolisation (PAE) is described as safe and effective. Aim of this study is to report our results, focusing on sexual outcomes (erectile and ejaculatory functions sparing) of PAE in patients suffering from bladder outlet obstruction (BOO) secondary to BPH.

Materials and methods: We prospectively enrolled and submitted to PAE subjects suffering from BOO secondary to BPH. All patients were not suitable for surgery or declined invasive approaches. All subjects were preoperatively and postoperatively (3, 6, 12 and 18 months after) evaluated by urinary flowmetry, post voiding residual volume, prostate volume, serum PSA levels, International Index of Erectile Function, International Prostate Symptom Score and QoL scores.

Results: PAE was performed in 147 patients (mean age 72.5 y.o.). PAE was technically successful in all patients. The procedure lasted a mean time of 94.3 minutes, with a mean fluoroscopic time of 42.5 minutes. Twelve months follow-up data were available for all patients, while 126 patients (85\%) completed the 18 months follow up. At 12 months follow up, the mean IPSS and QoL scores significantly decreased, and all the objective parameters (mean Qmax, PVR and prostate volume) reported a significant improvement. A total of 130 patients (88.5\%) at 12 months reported the antegrade ejaculation preserved, and a slight not significant improvement of IIEF scores. The 18 months after PAE outcomes confirmed the significant improvement of all the variables evaluated (even for PSA values and IIEF scores). No major complications occurred.

Conclusions: Our results evidence prostate artery embolisation as highly feasible and safe procedure with interesting outcomes. In particular, in our study PAE reported promising results in preserving antegrade ejaculation and erectile function. Our data are in line with the literature, confirming how PAE reduces obstructive symptoms in $\mathrm{BPH}$ patients not suitable or refusing standard surgical approaches.

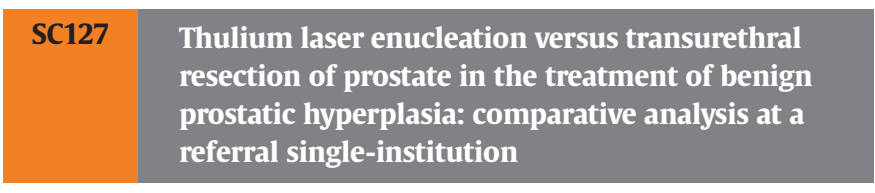

R. Bertolo, M. Vittori, C. Cipriani, F. Maiorino, V. Iacovelli, F. Petta, M. Panei, S. Travaglia, P. Bove (Roma)

Introduction: To compare the outcomes of thulium laser enucleation of prostate (ThuLEP) versus those of transurethral resection of prostate (TURP) in the treatment of benign prostatic hyperplasia (BPH).

Materials and methods: From September 2018 to February 2020, baseline, perioperative and postoperative data of consecutive patients scheduled for endoscopic surgery for BPH at our Institution were collected on a dedicated database. Specifically for the purpose of the analysis, patients were stratified into two groups according to the treatment received (ThuLEP vs. TURP). Indication was dictated by the referral urologist scheduling the surgery. While TURPs were performed by urologists skilled in the technique, ThuLEPs were performed by surgeons within the learning curve. Comparative analysis of perioperative and postoperative outcomes included the irrigation time, the catheterization time, the complications, and the eventual improvement in the international prostate symptom score (IPSS), quality-of-life (QoL) score, maximum flow rate (Qmax), and postvoiding residual volume (PVR) in both groups, at 1 and 3 months after surgery.

Results: 286 and 234 patients underwent ThuLEP and TURP within the time span considered,respectively. Groups were comparable at baseline. No significant differences were observed in the operative time $(55.7 \pm 30.4$ vs $60.2 \pm 16.6$ minutes, ThuLEP versus TURP, respectively, $\mathrm{p}=0.2$ ) but a significantly lower hemoglobin drop favored ThuLEP $(-0.6 \mathrm{vs}-1.6 \mathrm{~g} / \mathrm{dL}, \mathrm{p}=0.02)$. Nevertheless, this did not translate into differences between the groups in terms of irrigation time, catheterization time, and hospital stay. Within the 3-months follow-up, IPSS, Qmax, QoL, and PVR significantly improved in both groups without significant differences between ThuLEP versus TURP. No severe complications were reported; however, the occurrence of transient postoperative urge incontinence was higher in the ThuLEP group.

Conclusions: In our hands, both ThuLEP and TURP appear to be safe and effective in the treatment of BPH. ThuLEP could tend to reduce intraoperative blood loss but leads to more frequent postoperative irritative symptoms. Such finding could be related to the ongoing learning curve.

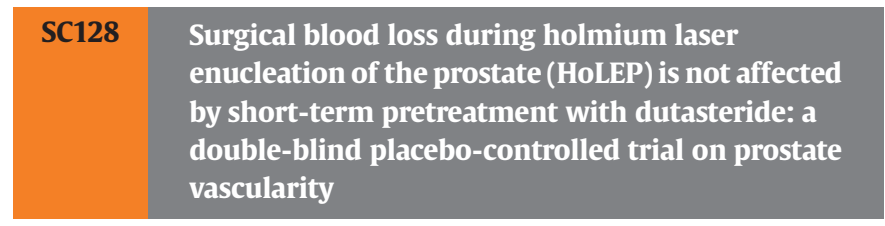

G. M. Busetto, F. Del Giudice, M. Maggi, D. Romagnoli, D. D’Agostino, A. Del Rosso, P. Corsi, M. Colicchia, G. Lucarelli, M. Ferro, O. de Cobelli, A. Sciarra, E. De Berardinis, A. Porreca (Roma)

Introduction: Five $\alpha$-reductase inhibitors (5ARIs) are able to reduce prostate volume and are a useful treatment for reducing perioperative bleeding during prostate surgery. Holmium laser enucleation of the 